(C) Masson, Paris, 1980.

\title{
Parasites de Chéloniens en Afrique Intertropicale
}

\author{
Polystomoides nabedei n. sp. (Monogenea, Polystomatidae),
}

parasite de la vessie urinaire de la tortue d'eau douce

Pelomedusa subrufa Lacépède, 1788 (Chélonien, Pelomedusidae).

\author{
par Sim-Dozou KULO *
}

(Collaboration technique : Marie-Thérèse ALMERAS **)

* Laboratoire de Parasitologie, Ecole des Sciences, Université du Bénin, B.P. 1515, Lomé (Togo)

** Département de Biologie animale, Université, F 66025 Perpignan Cedex

RESUME. Description de Polystomoides nabedei n. sp., parasite de la vessie urinaire de la Tortue d'eau douce Pelomedusa subrufa Lacépède, 1788 au Togo.

P. nabedei est voisin de Polystomoides chabaudi Euzet et Combes, 1965 décrit à Madagascar et signalé en Ouganda par Tinsley (1973) chez le même hôte, mais s'en distingue par les crochets de la paire externe nettement plus longs, les crochets de la paire interne montrant une insertion de la garde très voisine de la pointe (alors qu'elle est à la mi-longueur du crochet chez $P$. chabaudi), l'extension des vitellogènes vers l'arrière nettement plus importante.

Parasites of Chelonians in intertropical Africa : Polystomoides nabedei n. sp. (Monogenea, Polystomatidae), a parasite of the bladder of the freshwater tortoise Pelomedusa subrufa Lacépède, 1788 (Chelonian, Pelomedusidae).

SUMMARY. Description of Polystomoides nabedei n. sp., a parasite of the bladder of the freshwater tortoise Pelomedusa subrufa Lacépède, 1788 in Togo.

P. nabedei is similar to Polystomoides chabaudi Euzet et Combes, 1965 described in Madagascar and reported from Uganda by Tinsley (1973) in the same host, but differs in that the hooks of the external pair are longer, the hooks of the internal pair show an insertion of the hilt very close to the point (while it is found to half the length in $\mathrm{P}$. chabaudi), the vitellogenes are more developed in the post-testicular area.

Accepté le 4 avril 1980. 
En août 1978, nous avons récolté dans une mare du Nord-Togo 16 Tortues d'eau douce de l'espèce Pelomedusa subrufa. Dix d'entre elles abritaient dans leur vessie urinaire un Monogène Polystomatidae dont nous donnons la description ci-après.

\section{Polystomoides nabedei n. sp.}

Hôte : Pelomedusa subrufa Lacépède, 1788 *.

Localité : Mare temporaire de Siborototi (Dapaong, Togo).

Matériel de description : 10 individus colorés au carmin de Grenacher et montés en préparations in toto; 7 individus débités en coupes sériées transversales ou longitudinales. Type déposé au Muséum National d'Histoire Naturelle de Paris, sous le numéro TJ 63.

\section{Mensurations.}

Les mensurations du corps et des principaux organes de 10 exemplaires et les moyennes correspondantes sont données dans le tableau $I$.

Corps.

Les parasites (fig. 1 a) ont un corps allongé et aplati dorso-ventralement, de couleur blanchâtre. La silhouette générale est celle d'un Polystomatidae ; l'emplacement des ouvertures vaginales n'est marqué par aucun mamelon latéral mais on peut les voir en position latéro-ventrale sur l'animal vivant.

\section{Hapteur.}

Le hapteur occupe environ le quart de la longueur totale de l'animal. Il porte trois paires de ventouses dont les diamètres, d'après les mesures effectuées sur dix exemplaires, sont pratiquement identiques pour un individu donné.

L'armature du hapteur comprend deux paires de crochets et huit paires de crochetons larvaires à ogives.

Les grands crochets de la paire externe (fig. 1 b) ne possèdent ni manche ni garde et leur lame courbe est terminée en pointe fine.

Les crochets de la paire interne (fig. 1 c) nettement plus petits, possèdent au contraire un manche et une garde; le manche est fin et rectiligne; la garde courte est insérée aux trois quarts de la longueur du crochet en partant de la base ; la pointe est fortement recourbée.

Les crochetons larvaires pourvus d'un manche et d'une garde sont tous semblables (fig. 1 d) et mesurent 23 à $24 \mu \mathrm{m}$ de longueur ; 2 paires sont interventousaires

(*) Nous remercions M. Roger Bour, du Muséum National d'Histoire Naturelle de Paris, qui a bien voulu déterminer l'espèce d'hôte. 


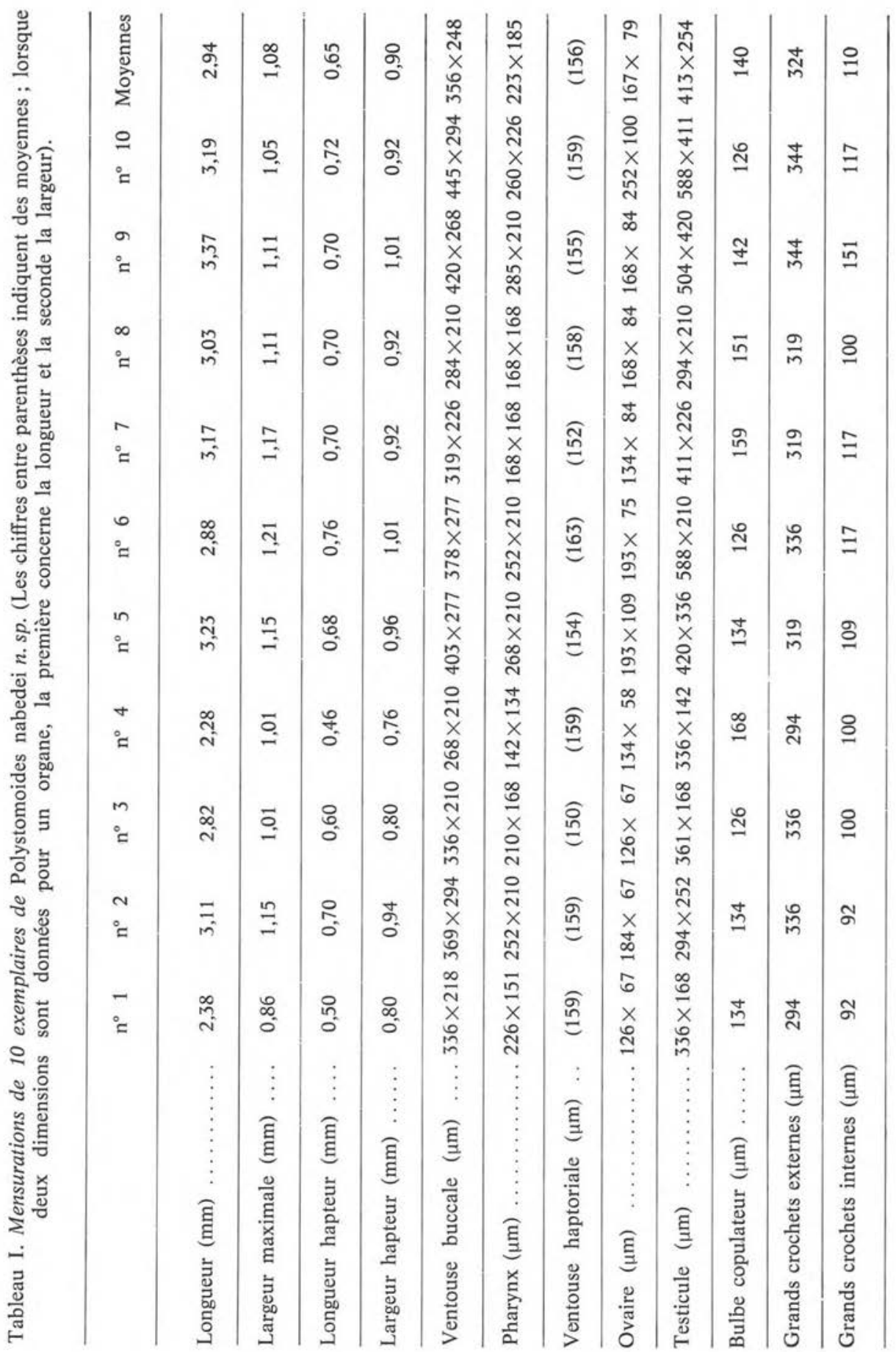




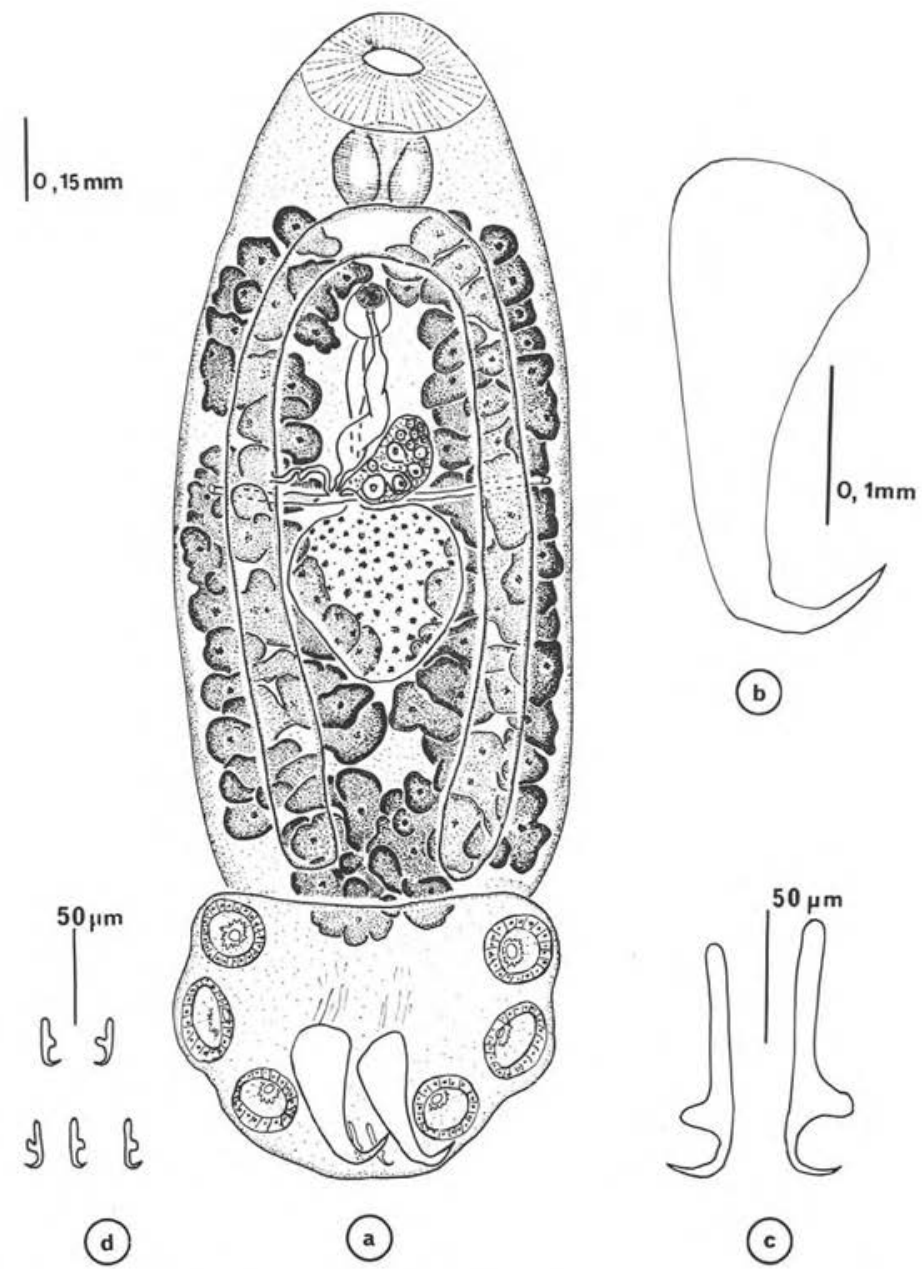

Fig. 1. Polystomoides nabedei n. sp. $a$ : individu in toto, vue ventrale; $b$ : grand crochet de la paire externe; $c$ : grand crochet de la paire interne; $d$ : crochetons.

postérieures, 3 paires interventousaires antérieures, les autres crochetons occupent le centre des ventouses.

Appareil digestif.

La bouche s'ouvre au fond de la ventouse orale, subterminale ventrale. Le pharynx est fortement musculeux; l'œsophage est presque inexistant; le tube digestif se divise en deux branches latérales subrectilignes qui se terminent au niveau du bord antérieur du hapteur sans jamais se fusionner. 
Appareil génital mâle.

L'unique testicule intercæcal, subsphérique, est situé à la mi-longueur de l'animal. Le canal déférent se renfle dorsalement à l'utérus en une vésicule séminale qui débouche dans le bulbe copulateur; ce dernier (fig. 2 a) à double paroi musculaire, est traversé par un canal éjaculateur, aboutissant à un cirre court qui est situé, au repos, en arrière d'une couronne de 36 à 39 épines génitales de 42 à $46 \mu \mathrm{m}$ de longueur, disposées autour de l'orifice mâle. Le pore génital mâle médio-ventral est situé en arrière du niveau de la bifurcation intestinale.

Appareil génital femelle.

L'ovaire, oblong, relativement petit, occupe une position immédiatement antérotesticulaire; il est paramédian à droite ou à gauche. La zone de multiplication des ovogonies se présente comme un prolongement antérieur de la masse principale de l'ovaire.

Sur le trajet de l'oviducte se situe un carrefour d'où part le canal génito-intestinal et où débouche le vitelloducte médian.

Le canal génito-intestinal forme des boucles pour aller se jeter dans le cæcum en avant ou en arrière du niveau du vitelloducte transverse.

L'oviducte débouche dans un ootype de grande taille qui devrait être en fait appelé ootype-utérus dans la mesure où c'est à la fois le lieu de la formation de l'œuf et celui où il séjourne brièvement avant d'être pondu; sa paroi est tapissée de grosses cellules bien individualisées. A la base de l'ootype se trouve la glande de Mehlis.

Sur l'un de nos exemplaires (fig. 2 b), nous avons pu saisir le processus de formation du zygote : au centre de l'ootype se trouve un ovocyte dans lequel pénètre un spermatozoïde et près de lui une première cellule vitelline, tandis que dans l'oviducte se trouvent d'autres cellules vitellines qui vont venir entourer le zygote.

Lorsque l'œuf est formé, il est de couleur jaune doré, operculé, et mesure 275 à $332 \mu \mathrm{m}$ de long.

Les glandes vitellogènes sont constituées par de nombreux follicules formant deux aires latéro-dorsales aux cæcums qu'ils dissimulent plus ou moins complètement; leur extension va du niveau postérieur du pharynx jusqu'au bord antérieur du hapteur où les follicules droits et gauches convergent et se confondent. La figure 3 montre la disposition, principalement dorsale, des vitellogènes à différents niveaux du corps.

Le pore génital femelle est situé juste en arrière de l'orifice du bulbe copulateur.

\section{Biologie.}

Le développement des Polystomoides n'a pas été étudié à ce jour et la question reste posée des modalités précises de la transmission et des possibilités de cycle interne. 

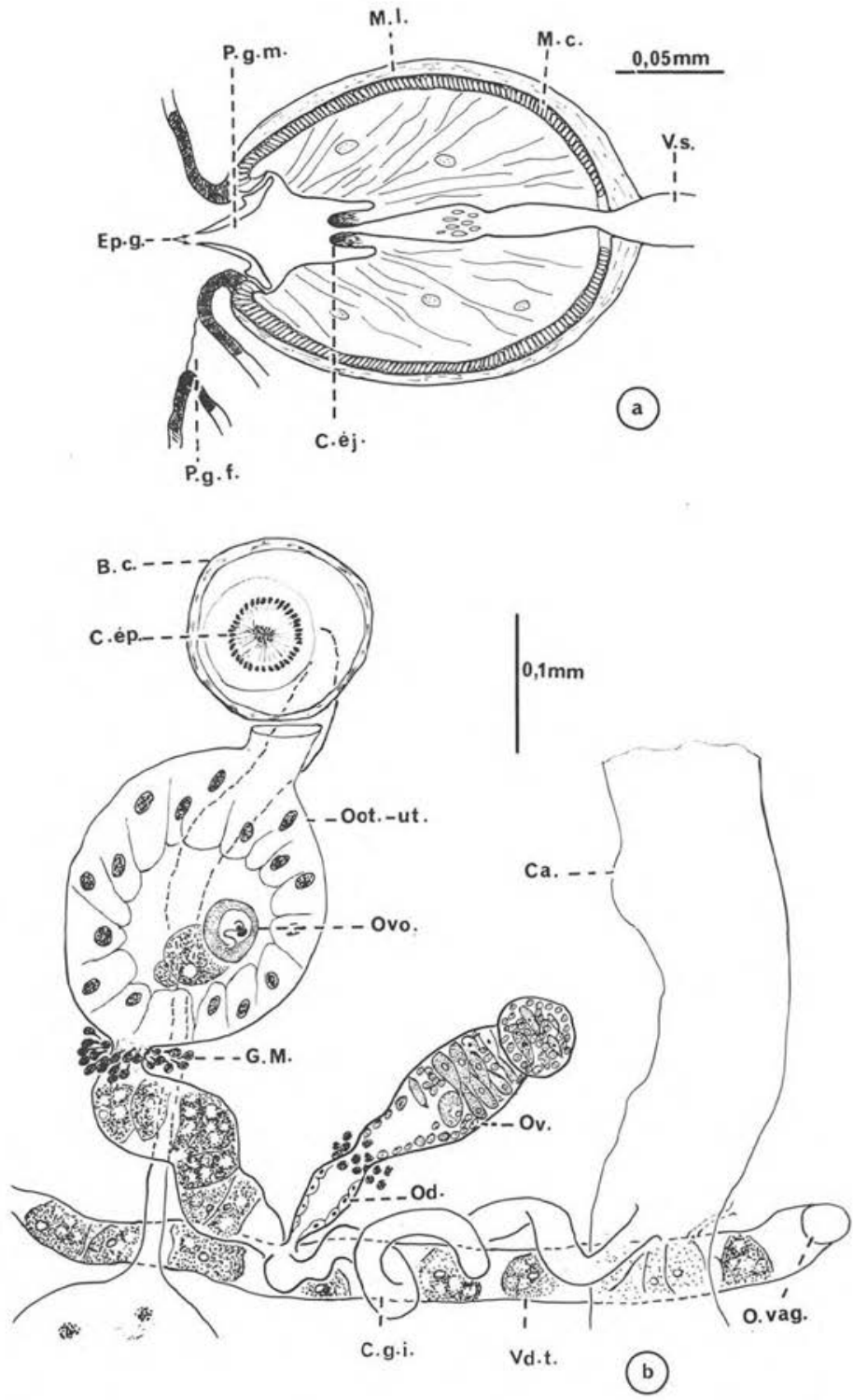

Fig. 2. P. nabedei. Anatomie de l'appareil génital. $a$ : bulbe copulateur en coupe longitudinale; $b$ : complexe génital femelle d'après une préparation in toto. 


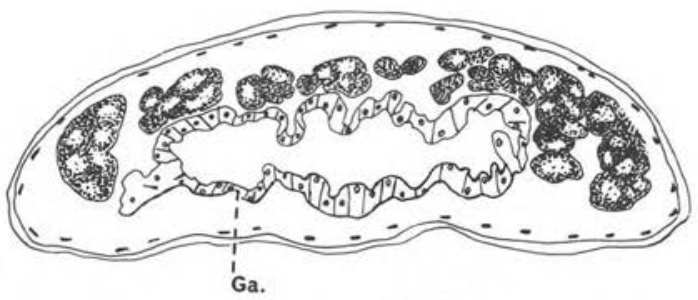

(a)

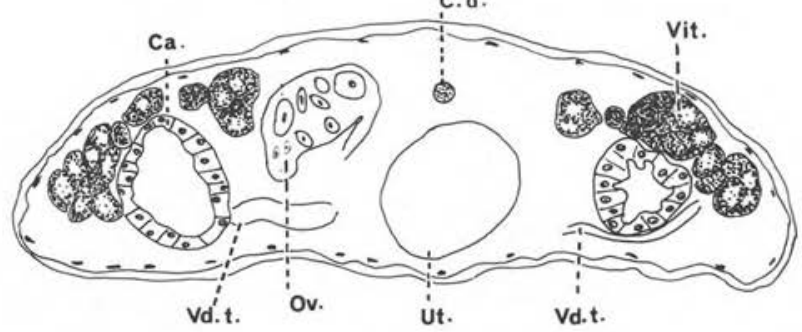

(b)

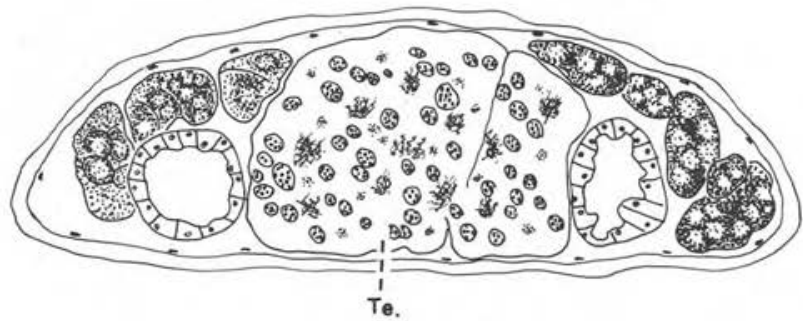

(c)

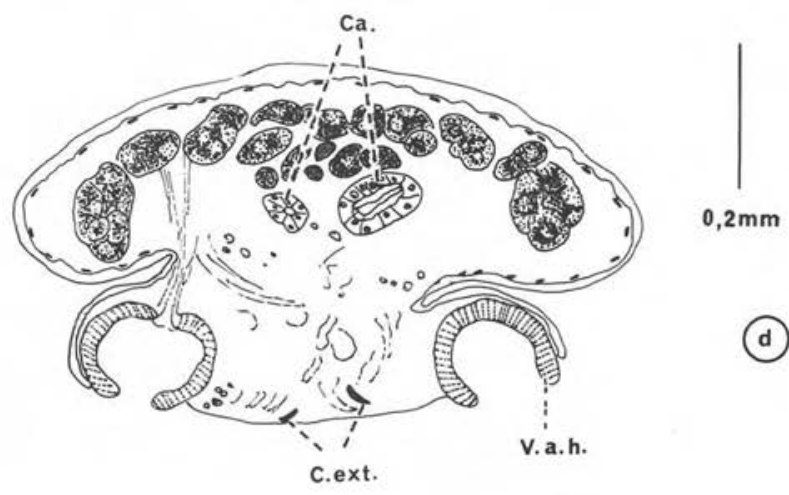

3.

Fig. 3. P. nabedei. Extension des vitellogènes à différents niveaux d'après des coupes transversales. 
Nous avons fait plusieurs observations qui nous paraissent apporter des éléments de réponse :

- dans l'utérus des parasites adultes, il existe souvent un œuf contenant une larve complètement formée,

- les œufs obtenus par dilacération des adultes fournissent habituellement des larves ciliées mais dans deux cas nous avons vu sortir de l'œuf une larve dépourvue de toute cellule ciliée,

- les coupes sériées pratiquées au niveau des uretères nous ont permis d'y découvrir des larves et des adultes, parfois (fig. 4) au niveau du rein lui-même.

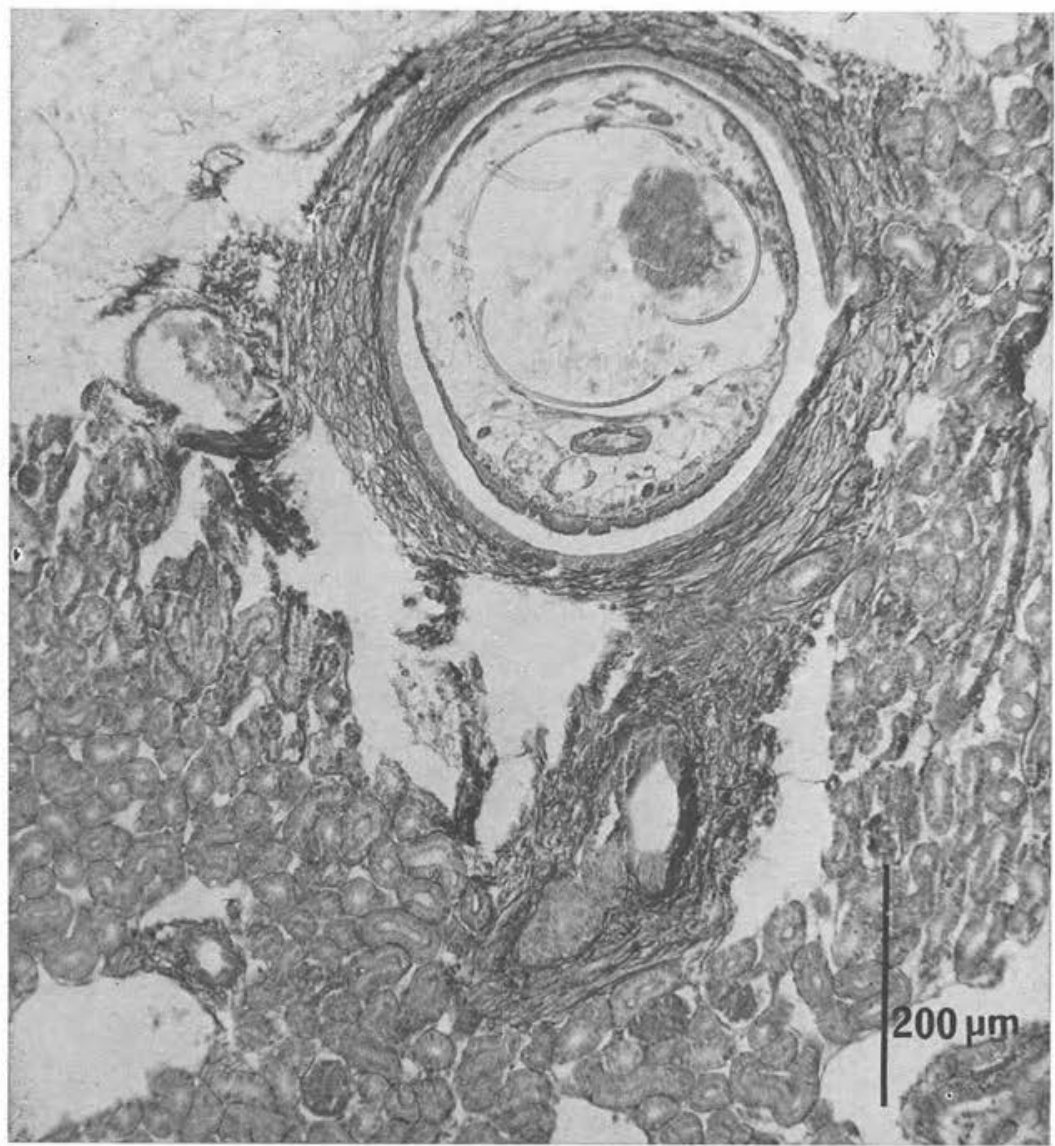

Fig. 4. Coupe de rein de Pelomedusa subrufa montrant un Poiystomoides adulte et ovigère dans l'uretère. 
Ces observations nous font penser qu'on peut envisager pour $P$. nabedei un cycle biologique comportant des similitudes avec celui du parasite d'Amphibien Eupolystoma alluaudi, étudié par Combes et al. (1973), Salami-Cadoux (1979), Fournier et Combes (1979) : développement des œufs dans l'utérus, existence de deux types de larves, inflation de la population vésicale par cycle interne, développement des larves dans les uretères. Cette similitude serait parfaitement corrélée avec la similitude dans la biologie de l'hôte, les mares où nous avons récolté les Tortues étant entièrement asséchées une partie de l'année.

\section{Discussion}

L'ensemble des caractères morphologiques et anatomiques de l'espèce que nous décrivons permettent de la ranger dans le genre Polystomoides Ward, 1917.

Le genre Polystomoides comprend actuellement 20 espèces : 18 espèces recensées par Combes (1976) auxquelles s'ajoutent Polystomoides bourgati Combes et Kulo, 1978 et Polystomoides platynotae Combes et Rohde, 1980. Par rapport aux deux sousgroupes de Polystomoides distingués par Knoepffler et Combes (1977) nos exemplaires, avec leurs grands crochets de longueur nettement supérieure aux diamètres ventousaires, confirment la relation entre le fort développement des grands crochets et l'habitat vésical.

Parmi l'ensemble des espèces du sous-groupe vésical nos spécimens se distinguent facilement des huit espèces récoltées en Eurasie, et de $P$. bourgati récoltée en Afrique.

Par contre, ils présentent une ressemblance certaine avec Polystomoides chabaudi Euzet et Combes, 1965 décrit chez le même hôte à Madagascar et signalé - toujours chez P. subrufa - en Ouganda par Tinsley (1973).

Nous avons pu comparer nos exemplaires avec le type et les cotypes de $P$. chabaudi.

Malgré une parenté évidente, il n'est pas possible d'attribuer nos spécimens à l'espèce $P$. chabaudi à cause des différences suivantes :

- crochets de la paire externe (fig. $5 a, b, c, d$ ) nettement plus longs que ceux de $P$. chabaudi (fig. $5 e, f$ ) pour des animaux de dimensions comparables;

- crochets de la paire interne montrant une insertion de la garde voisine de la pointe alors qu'elle est à la mi-longueur du crochet chez $P$. chabaudi;

- extension des vitellogènes vers l'arrière nettement plus importante que chez $P$. chabaudi, les follicules vitellins dépassant le niveau des cæcums et occupant la zone intercæcale post-testiculaire.

En conséquence nous considérons que nos exemplaires appartiennent à une espèce nouvelle pour laquelle nous proposons le nom de Polystomoides nabedei en hommage à notre maître le Pr Pakaï Nabédé, de l’Université du Bénin. 


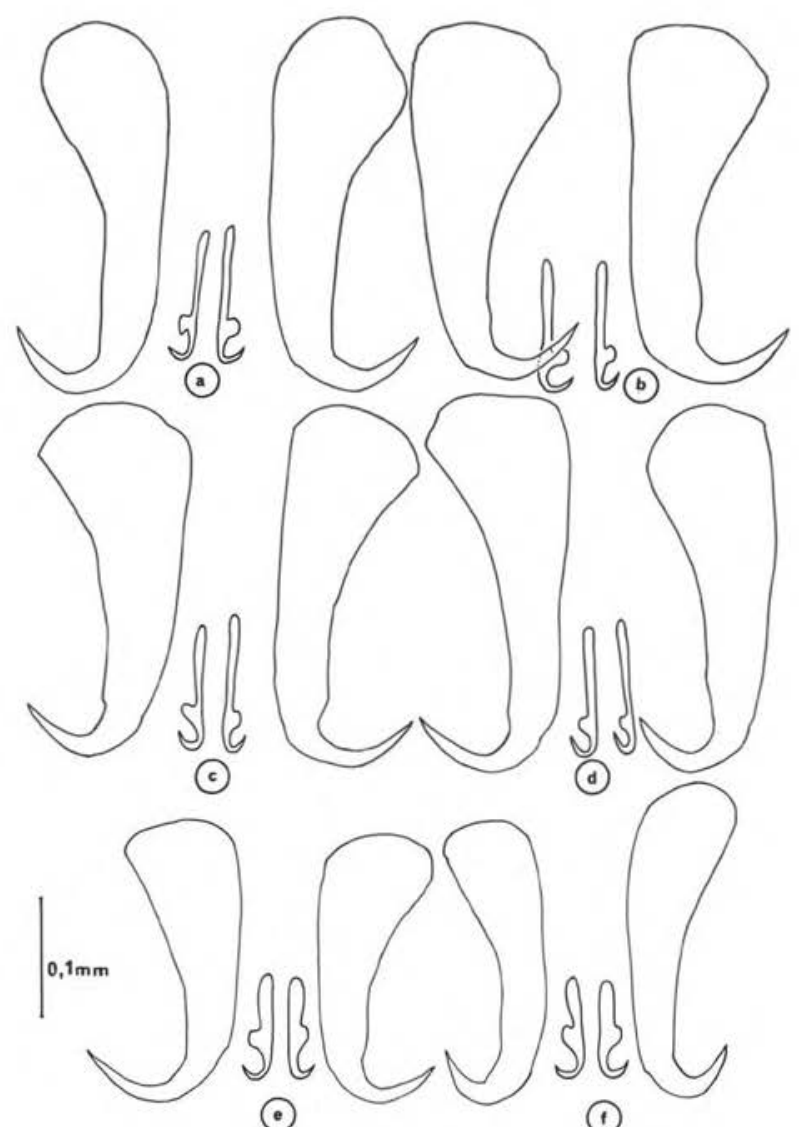

(e)

Fig. 5. Grands crochets de P. nabedei (a, b, c, d) et de P. chabaudi (e, f)

Il est intéressant de souligner que $P$. chabaudi et $P$. nabedei sont parasites de la même espèce d'hôte mais dans des régions très différentes de son aire. On peut penser que la faible mobilité des Tortues et l'importance de l'aire géographique ne permettent qu'un brassage génétique limité pour l'hôte comme pour le parasite et il semble que ceci ait entraîné une spéciation chez les Polystomoides mais non chez les Pelomedusa. Dans cette hypothèse $P$. chabaudi et $P$. nabedei seraient issus d'un ancêtre commun, parasite sur le même hôte.

L'examen des dessins de Tinsley (1973) pour $P$. chabaudi récolté en Ouganda nous fait d'ailleurs penser qu'il pourrait s'agir là d'une troisième espèce, parente de $P$. chabaudi et de $P$. nabedei. 


\section{Abréviations utilisées dans Les figures}

$\begin{array}{ll}\text { B.c. } & \text { : Bulbe copulateur } \\ \text { Ca. } & \text { : Cæcum } \\ \text { C.éj. } \quad \text { : Canal éjaculateur } \\ \text { C.d. } \quad \text { : Canal déférent } \\ \text { C.g.i. } \quad \text { : Canal génito-intestinal } \\ \text { C.ép. : Couronne d'épines } \\ \text { C.ext. : Crochets externes } \\ \text { Ep.g. : Epines génitales } \\ \text { Ga. : Gastroderme } \\ \text { G.M. : Glande de Mehlis } \\ \text { M.c. : Muscles circulaires } \\ \text { M.l. : Muscles longitudinaux }\end{array}$

Od. : Oviducte

O.vag. : Ouverture vaginale

Oot.ut. : Ootype-utérus

Ov. : Ovaire

Ovo. : Ovocyte

P.g.f. : Pore génital femelle

P.g.m. : Pore génital mâle

Te. : Testicule

Ut. : Utérus

V.a.h. : Ventouses antérieures du hapteur

V.s. : Vésicule séminale

Vit. : Vitellogène

Vd.t. : Vitelloducte transverse

Combes C.: Biogéographie mondiale des Monogènes Polystomatidae (en russe). In : Volume in memoriam Acad. B.-E. Bychowsky, 1976, 55-68.

Combes C., Bourgat R., Salami-Cadoux M.-L. : Biologie des Polystomatidae : le cycle interne direct chez Eupolystoma alluaudi (de Beauchamp, 1913). Z. Parasitenk., 1973, 42, 69-75.

Combes C., Kulo S.-D. : Polystomoides bourgati n. sp. (Monogenea, Polystomatidae), premier représentant du genre Polystomoides Ward, 1917 en Afrique occidentale. Rev. Zool. Afr., 1978, 92, 622-626.

Combes C., Rohde K.: Polystomoides platynotae n. sp. (Monogenea, Polystomatidae), parasite du Chélonien d'eau douce Notochelys platynota (Gray, 1834) en Malaisie. Vie Milieu, 1980, 28-29, $1 \mathrm{C}$ (sous presse).

Euzet L., Combes C.: Parasites des Chéloniens malgaches. Polystomoides chabaudi n. sp. (Monogenea) chez la Tortue d'eau douce Pelomedusa subrufa Lacépède, 1788. Ann. Parasitol., Hum. Comp., 1965, 40, 445-450.

Fournier A., Combes C.: Démonstration d'une dualité évolutive des embryons chez Eupolystoma alluaudi (Monogenea, Polystomatidae) et de son rôle dans la genèse du cycle interne. C.R. Acad. Sci. Paris, 1979, 289, sér. D., 745-747.

Knoepffler L.-Ph., Combes C. : Présence en Corse de Polystomoides ocellatum (Rudolphi, 1819), chez Emys orbicularis (L., 1758) (Chelonia, Emydidae). Considérations sur la répartition mondiale du genre Polystomoides. Vie Milieu, 1977, 27, 2 C, 221-230.

Salami-Cadoux M.-L.: Cycle et épidémiologie de Polystomatidae parasites de 1'Amphibien Bufo regularis Reuss au Togo. Thèse Univ. Perpignan, 1979, 1-393.

Tinsley R. C. : Observations on Polystomatidae (Monogenoidea) from East Africa with a description of Polystoma makereri, n. sp., Z. Parasitenk., 1973, 42, 251-263. 